\title{
Experiencia de gamificación en Secundaria en el Aprendizaje de Sistemas Digitales
}

\section{Gamification Experience in Secondary Education on Learning of Digital Systems}

\author{
Jesús Carlos Díez Rioja’, David Bañeres Besora², Montse Serra Vizern² \\ ' Institut Quatre Cantons, Barcelona, España. carlos.diez@4cantons.cat \\ 2 Universitat Oberta de Catalunya, Barcelona, España. \{dbaneres, mserravi\}@uoc.edu
}

\section{Resumen}

El uso del juego, utilizado siempre en edades tempranas para motivar el aprendizaje, ha sido frecuentemente estigmatizado en edades más avanzadas al considerarse una pérdida de tiempo. Sin embargo, en los últimos años el fenómeno de la gamificación se ha convertido en una tendencia metodológica con una gran presencia en las aulas. En este artículo, además de realizar un estudio del estado del arte en gamificación en primaria y secundaria y estudios universitarios, se describe, también, la experiencia llevada a cabo en un instituto de Barcelona donde se ha utilizado una aplicación implementada ad hoc para enseñar, de una forma lúdica, contenidos de sistemas digitales en el contexto de la asignatura de Tecnología Industrial del bachillerato. Los resultados de la experiencia y opiniones de los estudiantes que han sido positivas se recogen en este artículo.

\section{Palabras Clave}

Enseñanza secundaria; gamificación; Tecnología electrónica; Innovación educacional

\begin{abstract}
Games have always been used in order to motivate learning at early ages. Nevertheless, during teen years, playing games have often been stigmatized as a waste of time. Thus, the phenomenon of gamification has become recently a methodological trend with a relevant presence in the classroom. In this paper, an analysis of previous work in gamification is performed in primary and secondary education. Next, the experience carried out at a secondary school in Barcelona is described where a program has been implemented ad hoc to teach, in a playful way, contents of digital systems in the context of the Industrial Technology course in secondary school. The results of the experience and the students' opinion that have been positive are summarized in this paper.
\end{abstract}

\section{Keywords}

Secondary education; Gamification; Electronic engineering; Educational innovations 


\section{Introducción}

En la actualidad, los estudiantes de escuelas, institutos y universidades son nativos digitales. Han crecido utilizando las nuevas tecnologías y presentan nuevas maneras y actitudes ante el proceso de aprendizaje. Los docentes deben hacer frente a nuevos retos para adaptar este proceso a unas nuevas necesidades y preferencias, además de utilizar diferentes metodologías para conseguir un alumnado participativo, motivado e implicado en su propio aprendizaje.

Existen muchas metodologías, recursos e instrumentos que se han aplicado para motivar a este nuevo tipo de alumnos utilizando las TIC (Tecnologías de la Información y la Comunicación) como canal de comunicación y de aprendizaje: trabajo por proyectos, robótica o lenguajes gráficos de programación, son algunos ejemplos. Actualmente el uso de dispositivos digitales como ordenadores portátiles, tabletas o teléfonos inteligentes por parte del alumnado es imparable, como lo demuestran los últimos datos consolidados, del curso 2013-2014, proporcionados por la Subdirección General de Estadística y Estudios del Ministerio de Educación, Cultura y Deporte?.

Por eso, han aparecido diversas iniciativas que proponen aplicar metodologías y aprendizaje para que los jóvenes aprendan competencias como el pensamiento computacional o la abstracción de problemas. El objetivo no es que todos los jóvenes sean programadores, sino que desarrollen habilidades que les ayuden a resolver problemas de forma analítica como lo haría un informático. Iniciativas como TACCLE3 (García-Peñalvo, 2016) o INVENTA² ofrecen recursos que profesores de primaria y secundaria pueden usar en sus aulas. Pero, aunque recursos como estos ayudan a recoger y difundir metodologías y buenas prácticas, aún no es suficiente. Los profesores deben de innovar para desarrollar para sus alumnos nuevos recursos para las diferentes especialidades.

Es por ello que parece razonable pensar como metodología docente la gamificación ${ }^{3}$. Si se aplica una estrategia correcta al introducir un juego dentro de un contexto educativo, aprovechando principios de recompensa, estatus, interacción o competitividad, se pueden fomentar ciertas acciones del estudiante incentivando un comportamiento determinado.

En este artículo tratamos la introducción de la lógica combinacional dentro de la especialidad de la electrónica digital (o sistemas digitales) en bachillerato y para tener motivados a los alumnos se ha decidido utilizar técnicas de gamificación. Se ha desarrollado un juego ad hoc para la práctica

\footnotetext{
https://www.educacion.gob.es/educabase/menu.do?type=pcaxis\&path=/Educacion/Centros/Sice/Series\&file=pcaxis\&l=s0 Proyecto INVENTA de la Universitat Oberta de Catalunya para acercar la tecnología y la programación a niños entre 7 y 14 años: http://inventa.uoc.edu/inicio/

El término gamificación es un anglicismo que puede definirse como el uso de técnicas, elementos y dinámicas propias de los juegos y el ocio en actividades no recreativas con la finalidad de potenciar la motivación y de reforzar la conducta para solucionar un problema o alcanzar un objetivo.
} 
de estos conceptos y en este artículo se describe la experiencia realizada. Consideramos que dicha experiencia es interesante como metodología de aprendizaje tanto en secundaria como en asignaturas de fundamentos de Electrónica Digital en la universidad.

Este artículo se estructura de la siguiente forma. En la Sección 2 se realiza una revisión del estado del arte de la gamificación en ámbitos educativos y se describen una serie de experiencias seleccionadas por su carácter innovador principalmente en primaria y secundaria. Además, se analizan algunos casos donde la gamificación ha sido utilizada para la enseñanza de la lógica inherente en los circuitos digitales y las puertas y funciones lógicas. La Sección 3 describe el juego desarrollado y la experiencia docente se muestra en la Sección 4 con un análisis de los resultados obtenidos. Finalmente, las conclusiones y el trabajo futuro se presentan en la Sección 5.

\section{Gamificación, educación y TIC}

\subsection{Un poco de bibliometría}

Los juegos han acompañado a la humanidad desde la antigüedad. Yacimientos arqueológicos demuestran (Rubio, 2014) que el origen de algunos juegos de mesa se remonta hasta el año 5780 a. C. En Mesopotamia, en las tumbas reales de la ciudad de Ur, un arqueólogo inglés descubrió en 1922 un juego bautizado como "juego de las 20 casillas", de una antigüedad de unos 3000 años, utilizado desde la India hasta Egipto. Y en la Grecia clásica, por ejemplo, Aristóteles ya hablaba de la importancia del juego en el crecimiento y desarrollo de los niños y jóvenes. La relación entre el juego y la sociedad no es algo nuevo: desde los primeros que simulaban batallas, como el ajedrez (que era utilizado en la Edad Media para enseñar estrategia militar) hasta los videojuegos más modernos e hiperrealistas, todos ellos son consecuencia del tiempo en el que nacieron y se desarrollaron. De hecho, la mayoría de los juegos han nacido como un reflejo de la vida real.

A comienzos de los años 90 del siglo XX la industria del videojuego aceleró la investigación académica sobre los juegos. Estas investigaciones descubrieron los tipos de jugador, las motivaciones internas y los disparadores psicológicos de las conductas compulsivas. Y, como parecía previsible, el avance en el conocimiento de los juegos, junto con el uso de las nuevas tecnologías asociadas a técnicas de "engagement" propias de otros sectores, han acabado por llevar la gamificación al ámbito educativo. Según Capponeto, Earp y Ott (2014) la consolidación de la gamificación en las aulas, desde la educación primaria a la universitaria, es una realidad. En su trabajo, donde revisan y clasifican, atendiendo a diferentes criterios, 120 artículos sobre gamificación publicados en diferentes países entre los años 2011 y 2014, afırman que se trata de un fenómeno que está experimentando un rápido 
crecimiento y aseguran que la adopción de técnicas de gamificación entre los docentes tiene como objetivos fundamentales aumentar la motivación y la implicación del alumnado en las actividades relacionadas con el aprendizaje, haciéndolas, en definitiva, más atractivas, cautivadoras y, en última instancia, efectivas. Se trata de técnicas que no solo han sido adoptadas en diferentes materias o asignaturas, sino que también han servido para mejorar competencias transversales relacionadas con la capacidad de colaboración, la creatividad y el aprendizaje autónomo.

\section{Búsquedas en Google Scholar sobre gamificación}

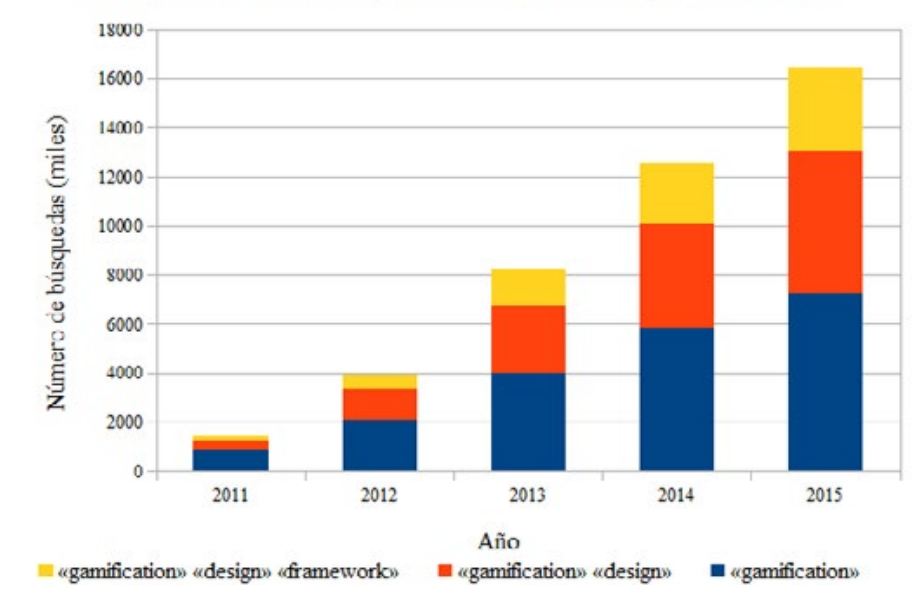

Figura 1. Búsquedas en Google Scholar sobre gamificación. Elaboración propia

El aumento de artículos científicos relacionados con la gamificación en todo el mundo es una realidad. Un buen ejemplo es el libro electrónico "Gamificação na educação" (Fadel, Batista, Ulbricht, y Vanzin, 2014), publicado en Brasil en el año 2014. A lo largo de casi 300 páginas y 10 capítulos, diversos especialistas explican conceptos relacionados con la gamificación en contextos educativos. Se analiza su uso como recurso para motivar al alumnado y se exploran qué mecanismos y elementos de los juegos son aplicables al diseño de un servicio, un producto o un proceso fuera de un contexto lúdico. Se reflexiona sobre la relación entre la gamificación y la "Teoría del Flow"4, ya que al capturar la gamificación la esencia de los juegos, las actividades gamificadas (donde aparecen metas claras, desafíos, sentimiento de control o pérdida de la noción del tiempo) proporcionan un estado de Flow. Se analiza la gamificación aplicada al "e-Learning", se reflexiona sobre la importancia de los hilos narrativos de los juegos, se investiga sobre la gamificación en las redes sociales para generar conocimiento con finalidades educativas o se proponen directrices para evaluar un juego.

Pero la revisión de trabajos sobre gamificación es una tarea relativamente joven y existen, aún, pocos marcos o "frameworks" teóricos de referencia, tal como explican Hamari, Koivisto, y Sarsa (2014). En este documento se revisan 24 estudios empíricos con el objetivo de argumentar si la gamificación 
realmente funciona. Estos documentos, localizados en diferentes bases de datos (Scopus, ScienceDirect, EBSCOHost, Web of Science, ACM Digital library, AISel, Google Scholar, y Proquest) se desarrollaron en diferentes contextos, siendo el ámbito educativo el más común (concretamente nueve trabajos). En los nueve se considera que la gamificación proporciona resultados positivos en el aumento de la motivación y la implicación del alumnado en actividades de aprendizaje. Sin embargo, se sugiere poner especial atención, ya que se consideran puntos débiles, en las dificultades en el proceso evaluador y en el propio diseño de los juegos.

En este sentido, debe tenerse presente que muchas soluciones gamificadas fracasan debido a que han sido concebidas mezclando elementos de aquí y de allá, sin un proceso de diseño claro y formal. La aplicación de un marco de referencia en lo que respecta al diseño es clave para garantizar el éxito de una solución gamificada, y la comunidad es consciente de ello: si se analizan el número de búsquedas en "Google Scholar" sobre los términos "gamification", "design" y "framework" los resultados demuestran el creciente interés hacia estos dos últimos términos, tal como puede observarse en la Figura 1.

Conscientes de esta falta de marcos de referencia, Mora, Riera, González, y Arnedo-Moreno (2015) efectúan una revisión de diferentes frameworks y proporcionan valiosos detalles que resultarán, sin duda, de gran utilidad para desarrolladores de soluciones gamificadas en diferentes ámbitos, incluido el educativo. Centran su atención en tres aspectos fundamentales: el estado del arte del proceso del diseño en gamificación, el análisis de la relación entre los procesos de diseño de los juegos y de las soluciones gamificadas y, en tercer lugar, la identificación y clasificación de los diferentes frameworks que existen actualmente.

Existen otros trabajos donde se describen iniciativas que han tenido una repercusión positiva en el aula. Llorens-Largo, Gallego-Durán, Villagrá-Arnedo, Compañ-Rosique, Satorre-Cuerda y Molina-Carmona (2016) desarrollan un juego para aprender el lenguaje Prolog donde resumen que lo importante es que el juego sea divertido, motive a los estudiantes, que haya progresión y haya retorno para aprender de los errores. Los mismos autores Llorens-Largo, Villagrá-Arnedo, Gallego-Durán, Satorre-Cuerda, Compañ-Rosique y Molina-Carmona (2016), a partir de esta contribución, proponen un modelo adaptativo de gamificación aplicable a cualquier plataforma online de aprendizaje basándose en los principios descritos en el anterior artículo.

\subsection{Estado del arte}

Son incontables las experiencias de gamificación en las aulas, llevadas a cabo en distintos niveles educativos, con el apoyo de las TIC. A continuación, se citan algunas, seleccionadas por su carácter innovador, ordenadas desde la enseñanza primaria hasta la universitaria. 
Eguía Gómez, Contreras Espinoza, y Solano Albajés (2015) llevaron a cabo un estudio del impacto sobre el uso de un juego digital denominado "Miquel Crusafont", centrado en descubrir al personaje y explicar su trabajo en paleontología. El área básica de trabajo era "Conocimiento del Medio Natural y Social", aunque había otras áreas relacionadas, como las matemáticas. Durante el desarrollo de la experiencia, los docentes destacaron que en la mayoría de actividades la habilidad del alumnado era superior a la del profesorado. Como aspecto por mejorar, destacaron la dificultad en planificar este juego dentro del aula por restricciones de currículo educativo y tiempo. Además, se consideró que son necesarias metodologías y formación específicas para que el profesorado pueda aplicar la gamificación en el aula.

Espinosa y Gómez (2012) destacan la potencialidad de la combinación de dispositivos móviles y la gamificación a través de videojuegos. Los autores (coautores del juego "Miquel Crusafont") afirman que los videojuegos en primaria contribuyen en el desarrollo de las habilidades sociales, aumentan el rendimiento escolar, las habilidades cognitivas, la motivación, el pensamiento complejo, y el trabajo en equipo.

Martín del Pozo (2015) también constata, en términos generales, la mejora en el aprendizaje del alumnado al realizar prácticas colaborativas en torno a un videojuego. La autora analiza ocho experiencias focalizadas de manera particular en el aprendizaje colaborativo. Demuestra que los videojuegos pueden ser utilizados para potenciar el aprendizaje y el trabajo colaborativo y que, en general, el alumnado consigue mejoras en el aprendizaje.

El uso del conocido videojuego "Minecraft"s en contextos educativos es analizado por Nebel, Schneider y Rey (2016). Actualmente, "Minecraft" está siendo utilizado como recurso educativo en todo el mundo en diferentes materias para enseñar, por ejemplo, geometría espacial, sostenibilidad, lengua y literatura, habilidades sociales, informática, diseño digital, gestión de proyectos o química. La empresa que desarrolla el videojuego, proporciona apoyo al profesorado y se ha desarrollado la versión educativa "MinecraftEdu", donde se integran herramientas de gestión para facilitar el trabajo de los docentes. Como consecuencia, se ha creado una comunidad mundial de profesores (MinecraftTeachers) donde estos comparten sus experiencias.

El proyecto SIGMA (Toda, do Carmo, Silva y Brancher, 2014) es un recurso en línea para ayudar a estudiantes de Brasil a alcanzar competencias matemáticas. Se trata de una plataforma interactiva dirigida al alumnado de primaria y secundaria con un doble objetivo: mejorar las competencias matemáticas del alumnado y facilitar el entrenamiento cuyo objetivo es: la "Olimpíada Brasileira de

\footnotetext{
5 Los aspectos creativos y de construcción de Minecraft permiten a los jugadores edificar construcciones creadas por cubos con texturas en un mundo 3D. En el juego también se incluyen otras actividades como, por ejemplo, la exploración, la recolección de recursos y el combate.
} 
Matemática das Escolas Públicas OBMEP". El sistema de gamificación se basa en la utilización de elementos motivadores, como por ejemplo puntos, insignias, bonus o certificados de nivel, con el objetivo de estimular a los estudiantes.

La buena simbiosis entre la gamificación y las TIC destaca en la experiencia llevada a cabo por Sanmugam, Abdullah, Mohamed, Aris, Zaid y Suhadi (2016) con un grupo de 29 estudiantes de un centro de secundaria del sureste de Malasia. En este caso, fueron las ciencias experimentales la asignatura elegida. Los elementos de gamificación utilizados en torno a estos contenidos fueron los puntos, las insignias y un ranking de posiciones. Los resultados mostraron que la combinación de tecnología y elementos propios del juego ayudaron en el cambio del comportamiento de los estudiantes hacia el aprendizaje.

El ámbito universitario, lógicamente, no es ajeno al fenómeno de la gamificación. En el libro electrónico "gamificación en aulas universitarias", Contreras (2016) ofrece una visión de la gamificación en las aulas universitarias españolas a través de algunas experiencias generadas por un grupo de expertos en diferentes centros de Madrid y Barcelona. Se enfatiza en diferentes aspectos del proceso de enseñanza-aprendizaje: la implicación y motivación del alumnado, la mejora que la gamificación puede suponer en el comportamiento y habilidades de los estudiantes, la mejora del proceso de evaluación en contextos gamificados, el fomento de la competencia amigable entre el alumnado y, por supuesto, la mejora en la adquisición de conocimientos.

Otro ejemplo del éxito de la gamificación en aulas universitarias es la experiencia que Lambruschini y Pizarro (2015) Ilevaron a cabo con estudiantes de la asignatura de tercer año "Gestión de Procesos" del grado de "Ingeniería de Sistemas Computacionales" de la Universidad de San Martín de Porres, en Perú. La educación en las universidades peruanas es compleja debido a que la mayor parte de los estudiantes trabaja y estudia de manera simultánea, especialmente en los últimos años de carrera. La mayor parte de ellos se limita a esforzarse en lo imprescindible para aprobar un determinado examen o entregar un trabajo sin profundizar, realmente, en el aprendizaje. Basándose en investigaciones sobre gamificación aplicada, se puso en marcha un sistema de premios denominado PEX ("Points of Experience"), donde a cada actividad le era asignada una determinada cantidad de puntos. Los resultados fueron determinantes, ya que la asistencia se incrementó del 60 al 86\%. La puntualidad experimentó un aumento impresionante, pasando del 10 al 79\% y la participación en los debates pasó del 15 al $47 \%$.

En el contexto de la asignatura "Multimedia Content Production" del máster "Information Systems and Computer Engineering" en el "Instituto Superior Técnico" de Lisboa, Barata, Gama, Jorge y Gonçalves (2013) analizaron la implicación de los estudiantes en cursos previos y comprobó la baja participación en la plataforma Moodle, la baja asistencia a clase y la falta de interés por los materiales facilitados 
(medida a partir del número de descargas, que era muy baja). Ante esta situación, y con la intención de aumentar la implicación y el interés del alumnado se decidió gamificar la asignatura. La metodología empleada consistía en premiar a los estudiantes a medida que finalizaban con éxito una actividad y en implementar una tabla con las clasificaciones donde se podían consultar los puntos obtenidos, ver las fotografías de los alumnos y el nivel alcanzado. Al finalizar la experiencia se comparó, utilizando diversas métricas, este curso gamificado con la versión no gamificada del curso anterior. Los resultados fueron muy positivos, ya que mostraban un claro aumento en el número de descargas de los materiales y, sobre todo, en la participación en los foros. El número de respuestas en los foros aumentó un 511\% y el número de nuevos hilos abiertos se incrementó un 845\%, lo cual denotaba no tan solo un espectacular incremento en la participación, sino también en la proactividad.

\subsection{Jugando con la lógica combinacional}

Una de las competencias que los estudiantes de grados relacionados con las telecomunicaciones, la informática o la electrónica deben adquirir es la capacidad de análisis y síntesis de circuitos secuenciales ycombinacionales. Los estudiantes debenenfrentarse desdesencillasimplementaciones de circuitos con puertas lógicas básicas hasta la intrincada anatomía de los circuitos integrados y, a menudo, el aprendizaje de la lógica combinacional y secuencial no resulta trivial. En este contexto, al alumnado, frecuentemente, le cuesta ver la diferencia entre la electrónica analógica y la digital a nivel práctico y se requieren de nuevas estrategias para que entiendan, por ejemplo, la finalidad de una tabla de verdad, o que las variables de entrada en un circuito pueden ser unos sensores y que la función de salida de la tabla puede significar la activación, o no, del receptor de un circuito.

Es escasa la literatura especializada que haga referencia al uso de la gamificación para enseñar lógica combinacional o fundamentos de sistemas digitales. No obstante, existen algunas experiencias interesantes. Por ejemplo, Wu, Zhu y Luo (2015) implementan un juego, denominado MineCircuit (basado en el ya citado MineCraft) para enseñar circuitos digitales a estudiantes de ingeniería electrónica. El diseño del juego, que se realiza a partir de un framework claro y bien definido, se basa en tres principios: guían a los estudiantes a través de retos de dificultad creciente (desde circuitos básicos a puertas CMOS o TTL de tres estados), combinan actividades de diseño de circuitos con aspectos del mundo real (por ejemplo, la distribución de agua desde un lugar más alto a uno más bajo se asimila a la manera de fluir de la corriente desde un nodo de potencial alto a uno de potencial más bajo) y, finalmente, la existencia de un hilo argumental desde el inicio del juego.

Otra experiencia de la gamificación aplicada a la enseñanza de circuitos digitales es la implementada en la Universitat Oberta de Catalunya, en el contexto de la asignatura de Fundamentos de Computación, impartida a distancia y donde las tasas de abandono son muy elevadas. Baneres (2016) explica 
la evolución del proceso. Uno de los recursos de la asignatura es una herramienta de aprendizaje autónomo denominada VerilUOC para practicar ejercicios relacionados con el diseño de circuitos. Con un profesorado sensibilizado por las tasas de abandono, se decidió potenciar el uso de VerilUOC. Se comenzó creando un espacio WIKI como manual de referencia y un aula de laboratorio común con acceso para todo el alumnado, pero, a pesar de que se observó un incremento en la actividad, su uso no se vio reflejado en los resultados académicos. Se decidió, entonces, introducir elementos de gamificación para potenciar, por un lado, el laboratorio como aula donde compartir dudas sobre la resolución de problemas y, por otro, potenciar la implicación del alumnado en la asignatura. Se comenzó con el diseño de dos retos relacionados con la resolución de un conjunto mínimo de problemas que proporcionaban unas recompensas extras en las notas finales, pero el impacto fue mínimo. Se rediseñaron y definieron de nuevo los retos, se mejoró la experiencia de usuario y en una última fase se creó una competición, donde el alumnado podía ver su posición en un ranking, con la entrega de medallas de oro, plata y bronce. Los resultados obtenidos mostraron una ligera mejoría en lo que respecta al abandono y el uso de la herramienta citada.

\section{La experiencia desarrollada}

El bachillerato, desde hace años, incluye tres asignaturas de marcado perfil tecnológico, como son el Dibujo Técnico, la Electrotecnia y la Tecnología Industrial. Se trata de asignaturas de modalidad, cursadas en su totalidad o en parte por estudiantes que quieren cursar ciclos formativos de grado superior o ingenierías. Concretamente, en la asignatura de Tecnología Industriall ${ }^{6}$ (y en menor medida en la asignatura de Electrotecnia), se introducen conceptos básicos relacionados con circuitos lógicos combinacionales y secuenciales, puertas y funciones lógicas y procedimientos de simplificación de funciones.

El instituto barcelonés Quatre Cantons del Poblenou, fundado el curso 2011-2012, se caracteriza por desarrollar un proyecto educativo que da cabida a un conjunto de actividades, de marcado carácter transversal, denominadas "Trabajo globalizado de propuesta externa". Se trata de propuestas de intervención que diversas entidades realizan al alumnado. Con una duración de unas 6 semanas y una dedicación de 8 horas semanales, el alumnado debe de situarse en la naturaleza del encargo o necesidad de la institución, idear la intervención, acción o propuesta y, de acuerdo con la entidad, Ilevarla a cabo. Debido a que los resultados son muy satisfactorios en la ESO, se ha querido prolongar la experiencia al bachillerato. En este sentido, el alumnado que cursa la asignatura de Tecnología Industrial 1 en primero de bachillerato ha recibido el encargo de organizar y tomar parte en la "Primera carrera de robots seguidores del distrito de Sant Marti", concebida, preferentemente, bajo el paradigma 
del hardware y software libre y para la cual necesitarán conocer algunos conceptos básicos de sistemas digitales. Por otro lado, el alumnado que cursa Tecnología Industrial 2 en segundo de bachillerato y pretende examinarse de la asignatura de Tecnología Industrial en las pruebas de acceso a la universidad debe resolver un ejercicio obligatorio sobre sistemas digitales que representa un 25\% de la calificación total de la prueba y que presenta siempre la misma estructura: se plantea un problema cuyas especificaciones pueden modelarse mediante tres variables binarias de entrada y una función lógica de salida; se solicita, en primer lugar, la tabla de verdad del sistema, posteriormente la función lógica simplificada y, finalmente, la representación del esquema de puertas lógicas correspondiente.

Ante esta situación, se estimó oportuno llevar a cabo una experiencia de gamificación con el objetivo de que los estudiantes aprendan y consoliden conceptos básicos de sistemas digitales y lógica combinacional de una forma lúdica. El diseño e implementación de la prueba de concepto se realizó a partir del estudio de los diferentes "framework" descritos en el estado del arte y a partir de una actividad colaborativa con los estudiantes, todo ello relacionado con el uso de los videojuegos. El resultado de esta actividad se describe en la siguiente sección.

\subsection{La lluvia de ideas}

Con el objetivo de diseñar un juego centrado en el usuario, a lo largo de siete días se realizaron un conjunto de actividades colaborativas, a modo de lluvia de ideas, con el alumnado de $1^{\circ}$ y $2^{\circ}$ de bachillerato que cursa las asignaturas de Tecnología Industrial. De estas sesiones se obtuvieron los siguientes resultados:

- Los videojuegos, en general, les satisfacen porque les hacen tomar decisiones en poco tiempo, y eso les gusta.

- Valoran las situaciones que plantean retos asequibles, pero que a la vez impliquen cierta complicación. Cuando fallan quieren seguir jugando para equilibrar la situación.

- En general, no consideran prioritarios los hilos argumentales, sino que lo trascendente son las oportunidades de volver a intentar lo que no se domina hasta conseguirlo. En definitiva, aunque un juego no "explique una historia demasiado interesante" se sienten cómodos en un entorno que controlan y gestionan y, si es posible, que tenga pocas reglas o normas. Las jugadoras y jugadores más ocasionales valoran que los juegos sean rápidos, intuitivos y que no exijan un alto grado de conocimiento.

- En los juegos también buscan el reconocimiento social dentro del grupo. Son muy competitivos, les gusta lucir los resultados que obtienen y ser admirados cuando ocupan las primeras posiciones en los rankings. 


\section{GAMIFICACIÓN on ol ámbito oducativo

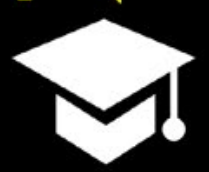

La gamificación consiste en utilizar técnicas, elementos y dinámicas propias de los juegos y el ocio en actividades no recreativas con la finalidad de potenciar la motivación y de reforzar la conducta para solucionar un problema o alcanzar un objetivo.

\section{Objetivos}

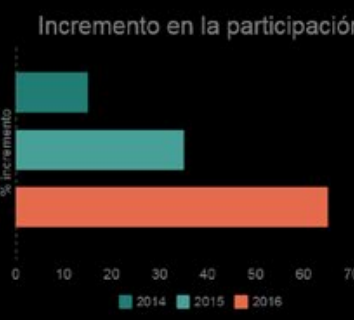

Aumentar la motivación y la implicación del alumnado

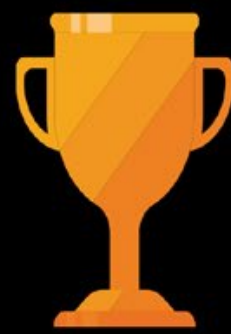

Mejorar los resultados y consolidar aprendizajes

Debilidades

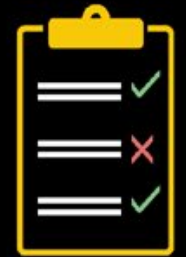

Dificultades en el proceso evaluado

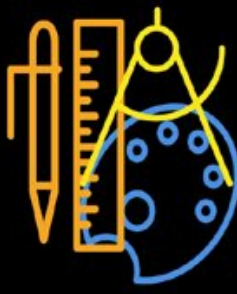

Ausencia de un diseño claro y formal

\section{Elementos comunes de soluciones gamificadas}

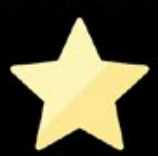
PUNTOS

Permiten comparar los logros de un alumno respecto al resto

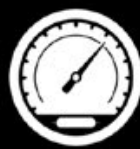

\section{NIVELES}

Animan al alumnado a progresary

superar nuevos retos

\section{Mo RANKINES \\ 11 Muestran la clasificación del \\ $\begin{array}{llll} & 1 & 3 & \text { alumnado }\end{array}$}

\section{(O) INSIGNAS}

Una forma visible de recompensa

$\left[\begin{array}{l}\text { RETOS } \\ \text { Animan a los estudiantes a completar } \\ \text { determinadas actividades }\end{array}\right.$ 
- Consideran imprescindible un feedback por parte del juego para saber en todo momento dónde están y qué posición ocupan respecto al resto de jugadores.

Finalmente, se puede afirmar que la relación que la mayoría tiene con los juegos acostumbra a ser vertiginosa, descubren un juego que les gusta, se "enganchan" y abusan durante un tiempo para después abandonarlo para siempre.

A partir de estas especificaciones se diseñó el juego en cuestión, tal como se describe en las siguientes secciones.

\subsection{Diseño del juego}

La infografía de la Figura 2 sintetiza el diseño del juego. En primer lugar, el objetivo principal del juego es aprender y consolidar los conceptos relacionados con la lógica combinacional. El juego debe motivar a los alumnos e implicarlos en el proceso de aprendizaje. Para el diseño del juego se ha tenido presentes dos aspectos. 1) La presencia de elementos comunes en las soluciones gamificadas como son los puntos de experiencia, los niveles, los rankings, las insignias y los retos de dificultad creciente. 2) En base a los resultados de la lluvia de ideas, se ha tenido en cuenta el tipo de alumnado a quien va dirigido, la experiencia de usuario mediante la utilización de las TIC, la información de su evolución en el proceso de aprendizaje y feedback continuo para mejorar las soluciones de los ejercicios. El uso de hilos narrativos era un objetivo a tener en cuenta que al final no se ha aplicado y se deja como trabajo futuro.

Finalmente, se ha optado por una metodología SCRUM de implementación, para obtener resultados a muy corto plazo y con diversas iteraciones donde los estudiantes de la asignatura han tenido un rol importante de "testers" para la implementación de una versión inicial del juego.

\subsection{Definición de actividad}

Antes de definir el juego y presentar la plataforma implementada es importante describir el tipo de actividad de aprendizaje del juego. Aunque, como se ha descrito anteriormente, el objetivo es "aprender de forma lúdica", no tenemos que olvidar que el alumno "debe aprender". Una actividad está formada por 4 ejercicios consecutivos. Un ejercicio consiste en la definición de una función lógica en formato de tabla de verdad o expresión booleana. El estudiante debe proponer un circuito combinacional con un conjunto de puertas predeterminadas que define el profesor en el momento de generar el enunciado. Actualmente se dispone de tres niveles de dificultad: inicial, intermedio y experto. La dificultad viene determinada por la complejidad de la función lógica que se tiene que 
diseñar, el número de entradas y el tipo de puertas disponibles para hacer el ejercicio. Debemos tener en cuenta que no se pide el diseño mínimo a dos niveles que nos podría dar un método simple de optimización como Karnaugh Maps, sino que el alumno debe utilizar las propiedades del álgebra de Boole para transformar la función en una que se pueda representar con las puertas que permite el ejercicio. Nótese que en esta primera versión del juego no se permiten bloques combinacionales complejos como multiplexores o codificadores.

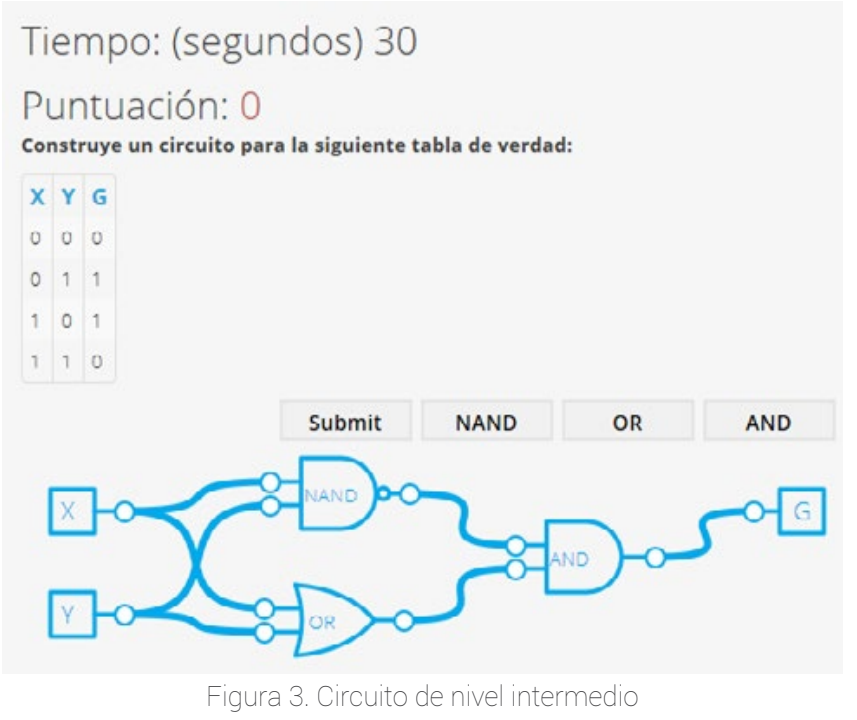

Un ejemplo de ejercicio de nivel intermedio se muestra en la Figura 3. La función que se pide es una simple XOR $\left(x y^{\prime}+x^{\prime} y\right)$, pero el ejercicio se complica al no permitir en este nivel ni puertas XOR ni negadores NOT combinadas con puertas AND y OR. Esta solución obtenida por un alumno, resuelve el ejercicio proporcionando la solución $(x y)^{\prime}(x+y)$ que es funcionalmente equivalente a la función XOR pedida en el enunciado.

Es importante que el estudiante tenga algún feedback en caso de error para poder mejorar el circuito diseñado. El juego devuelve un feedback en términos de valores para las señales de entrada de la función que son incorrectos en el circuito diseñado. El objetivo es que el alumno analice el error y mediante un proceso de prueba y error pueda obtener la solución correcta.

La Figura 4 muestra un diseño incorrecto para el ejercicio anterior. El circuito implementa la función $\left((x y)^{\prime}(x y)^{\prime}\right)^{\prime}$ que con diferentes propiedades del álgebra de Boole se obtiene la función simple xy. Como podemos observar, se devuelve un feedback de los mintérminos (o valores de las entradas) donde se encuentra el error. 


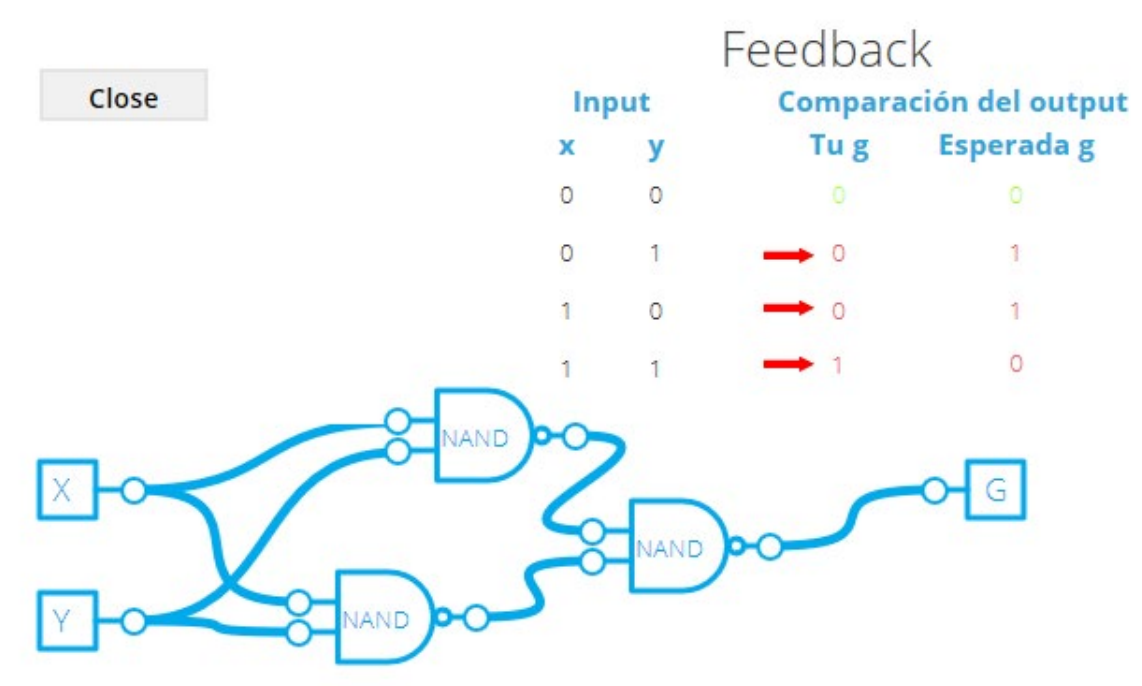

Figura 4. Feedback con las tablas de verdad del circuito propuesto y del requerido

\subsection{Interfaz del juego}

El juego se ha diseñado con las siguientes especificaciones.

- Juegos cortos de 4 ejercicios consecutivos.

- Definición de tres niveles de dificultad.

- Consulta de la clasificación.

- Consulta de la evolución personal.

- Configuración de la privacidad de datos.

\section{Puertas y circuitos lógicos

Menú principal
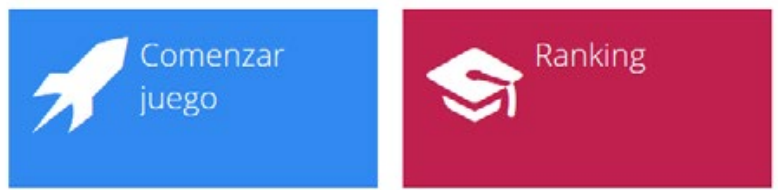

Evolución
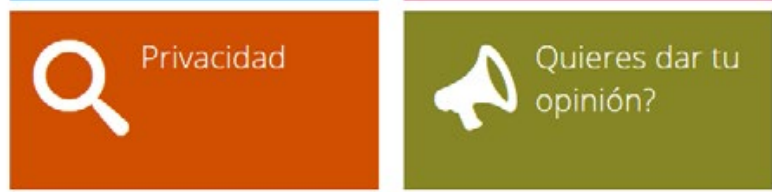

Figura 5. Menú principal 
El juego se ha implementado como una aplicación web que se puede observar en la Figura 5. El alumnado participante accede mediante un nombre de usuario y una contraseña. Desde el menú principal, el estudiante puede comenzar un nuevo juego, consultar la clasificación (ranking), su evolución personal y configurar la privacidad de datos.

Los diferentes niveles de juego siempre están activos. De esta forma, un estudiante puede probar desde el primer acceso los diferentes niveles de dificultad disponibles.

La mecánica del juego es: consiste en ir implementando circuitos lógicos con las puertas lógicas facilitadas con la particularidad de que hay un cronómetro activo. Si se implementa un circuito correcto, el usuario obtiene 100, 125 o 150 puntos (a los que se restan el número de segundos consumidos en implementar el circuito) dependiendo del nivel de dificultad. Nótese que, en caso de obtener puntos negativos como resultado de un ejercicio, se obtienen 0 puntos.

Si el circuito implementado es incorrecto, se restan 50, 75 o 100 puntos, (dependiendo de si el ejercicio corresponde al nivel inicial, medio o experto) y se proporciona la tabla de verdad del circuito propuesto por el alumno comparada con la requerida, de manera que se puedan observar las combinaciones incorrectas. Al superar un nivel determinado, la puntuación se almacena en el sistema.

Como hemos descrito, el juego tiene otras opciones de consulta. La opción "ranking" permite consultar la clasificación, donde aparecen las 15 máximas puntuaciones obtenidas por los miembros participantes. La opción "evolución personal" muestra el número de participaciones totales, la puntuación acumulada, la media de puntos obtenidos y su nivel (bit, byte o kilobyte) que depende del número de participaciones en el juego.

Es importante remarcar que inicialmente esta información no es pública (tan solo el profesor puede consultar el "ranking") y es decisión de los estudiantes mantener su privacidad o mostrar sus resultados al resto del grupo. Además, la configuración permite añadir un alias para que los alumnos que aun saliendo en el ranking quieran mantenerse en el anonimato.

\subsection{La implementación del juego}

La prueba de concepto se ha implementado utilizando tecnologías HTML5, PHP, MariaDB y JavaScript. En la parte cliente (el navegador) se ejecutan los scripts que comparan las soluciones propuestas con las tablas esperadas. Para ello se ha utilizado una librería desarrollada por Ville Karavirta ${ }^{7}$ y publicada bajo licencia MIT. En el servidor se alojan los ficheros php con los diferentes ejercicios propuestos 
y es desde donde se controlan, también, las validaciones de los usuarios y las variables de sesión. También se aloja en el servidor la base de datos relacional que contiene dos únicas tablas. En la tabla "clasificación" se almacenan el nombre de usuario, la fecha del ejercicio, la puntuación obtenida y el nivel correspondiente. En la tabla "privacidad" se guarda la decisión de hacer pública o no la puntuación obtenida (por defecto se define como privada) y el alias del alumno.

\section{Resultados de la experiencia}

La prueba se hizo en la asignatura de Tecnología Industrial en un grupo de 17 alumnos, de los cuales 13 son de Tecnología Industrial 1 y 4 de Tecnología Industrial 2. Los alumnos participaron previamente en la lluvia de ideas y por lo tanto conocían el objetivo del juego. Hemos de remarcar que previamente el profesor había explicado los conceptos teóricos de la lógica combinacional en clase. Por lo tanto, el juego sirvió, en cierta manera, para practicar de forma lúdica los conceptos teóricos desarrollados.

Uno de los grandes agentes motivadores en las soluciones gamificadas es el premio, ya sea ofrecido en forma de reconocimiento (como por ejemplo la aparición en las posiciones altas en la tabla de clasificación) o en forma de recompensa. En este sentido, antes de comenzar a jugar, los estudiantes fueron informados de la recompensa que obtendrían: "Quien complete, al menos, 4 juegos en cada uno de los tres niveles, obtendrá medio punto extra en la nota final del trimestre. También se premiará con un punto extra en la nota final a quien obtenga una de las tres puntuaciones medias más altas habiendo jugado, al menos, doce veces".

\section{Puertas y circuitos lógicos Evolución y participación}

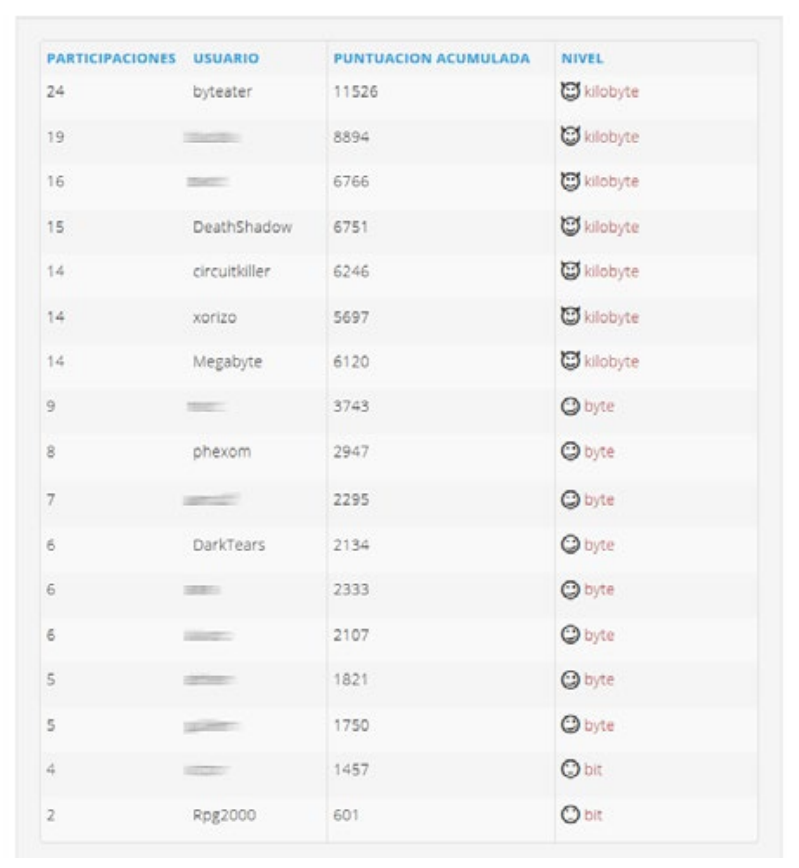


Después de tres semanas de juego el resultado del ranking se halla en la Figura 6. Siete estudiantes habían completado el número mínimo de participaciones necesarias para recibir la recompensa. De estos siete, una alumna prefirió centrarse en conseguir la media más alta y tan solo ha participado en los niveles intermedio y experto, por lo que recibió un punto de recompensa. Dos alumnos recibieron 1,5 puntos: medio punto por completar, al menos, cuatro juegos de cada nivel y un punto por haber registrado la segunda y la tercera posición del ranking. Finalmente, cuatro estudiantes recibieron medio punto de recompensa. El resto no recibió ninguna recompensa al no haber completado el número de ejercicios mínimos requeridos.

Nótese que los alumnos participaron como beta "testers" en las primeras versiones del juego y dieron su opinión para obtener la versión final. Gracias a sus habilidades encontraron posibles debilidades del juego y propusieron mejoras. Estas pruebas se hicieron durante el primer trimestre del curso 20162017. Al realizar la prueba final a finales del trimestre (diciembre 2016), algunos alumnos renunciaron a la recompensa, ya que los ejercicios que ofrecía el juego eran los mismos y ya no les motivaba. Por lo tanto, una mala planificación de la prueba final comprometió los resultados de la experiencia. De todos modos, este resultado se debe valorar en base a los comentarios previos de los alumnos en la Iluvia de ideas. Muchos alumnos se sintieron motivados por el juego durante el test de la aplicación. Se engancharon al juego durante las diferentes pruebas, pero durante la valoración final ya no les motivaba para continuar utilizándolo. Por lo tanto, los alumnos se comportaron tal como se definieron en un principio.

\subsection{Valoración de los estudiantes}

Para conocer la opinión de los estudiantes se implementó un cuestionario formado por un primer bloque de preguntas cerradas de elección única donde la valoración de las mismas se realizó utilizando la escala de Likert del 1 al 5 (siendo 5 el valor que corresponde a estar totalmente de acuerdo) y un segundo bloque formado por seis preguntas abiertas. El primer bloque se dividió, a su vez, en tres partes. En la primera se perseguía su opinión sobre el juego "Aprende lógica combinacional jugando" mediante preguntas relacionadas con la jugabilidad, la estructura, el aspecto, etc. La segunda parte estaba centrada en averiguar el estado de ánimo de los estudiantes mientras juegan. La tercera, finalmente, estaba concebida para valorar la opinión de los estudiantes respecto a la consolidación de contenidos de lógica combinacional. Las preguntas abiertas permitieron al alumnado expresar su opinión sobre los aspectos positivos o negativos del juego, describir los problemas técnicos que pudieron aparecer, sugerir alguna mejora o explicar el motivo de mantener (o no) el anonimato en las listas de clasificación.

La encuesta la respondieron 10 alumnos (58\% de participación). Es un valor suficientemente elevado 
para extraer conclusiones y analizar las opiniones iniciales sobre la jugabilidad del juego. Para reducir la extensión del análisis del cuestionario se ha optado por indicar conclusiones generales de la opinión de los alumnos.

En cuanto al juego "Aprender lógica combinacional jugando" lo consideran entretenido (73\%), fácil de jugar (80\%), de aspecto agradable (70\%), con un número de niveles adecuado (75\%) y que está bien organizado (90\%). Al parecer, el paso de los segundos del cronómetro hace que un porcentaje elevado se sienta presionado (75\%), aunque no tienen, en general, un sentimiento de frustración cuando fallan (30\%). También afırman que han aprendido con el juego (78\%), cumpliéndose sus expectativas, y que el feedback proporcionado les ha resultado de utilidad (78\%).

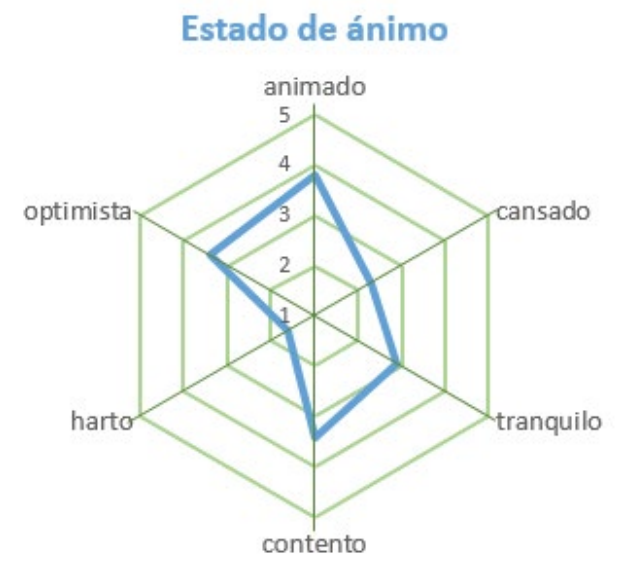

Figura 7. Estado de ánimo de los estudiantes según la encuesta

El análisis del estado de ánimo se puede observar en la Figura 7. Mientras jugaban, según sus palabras, se han sentido animados, contentos, tranquilos y optimistas. Los sentimientos relacionados con haberse cansado y sentirse hartos no tienen una valoración muy alta.

Casi la totalidad del alumnado que ha respondido al cuestionario cree que el juego les ha ayudado a consolidar los contenidos básicos de la lógica combinacional (85\%) y han entendido que las tablas se pueden sintetizar en circuitos lógicos (93\%). Han entendido cómo aplicar teoremas del álgebra de Boole y leyes de De Morgan para resolver algunos ejercicios (78\%) y han entendido que una función lógica, una tabla de verdad y un circuito lógico son tres maneras diferentes de representar la misma información (95\%).

Respecto a las preguntas abiertas, destacan la jugabilidad y la dificultad incremental en los niveles como aspectos positivos del juego. Han detectado algunos problemas técnicos, como la necesidad de ser muy precisos a la hora de realizar las conexiones entre las entradas y salidas de las puertas lógicas y han apuntado algunas mejoras de diseño, como por ejemplo la posibilidad de consultar el número de veces que han jugado cada nivel y la puntuación obtenida en cada caso (actualmente solo pueden consultar las 15 mejores puntuaciones y el número total de veces que han completado el juego 
con la puntuación acumulada). Otro aspecto a mejorar, consiste en introducir cierta aleatoriedad. En este sentido, sugieren que dentro de un determinado nivel, exista la posibilidad de jugar diferentes ejercicios en diferentes sesiones. Finalmente, en lo que respecta a la privacidad, la mayor parte prefiere publicar sus resultados y poder comparar su rendimiento y puntuación con la del resto del grupo, aunque sea con un alias.

\section{Conclusiones}

Los cambios en la forma de aprender en primaria y secundaria son una realidad. Mientras el pensamiento computacional no esté en el currículo escolar de forma obligatoria, los profesores deben usar metodologías innovadoras para introducir esta competencia necesaria para los jóvenes. En este artículo se ha presentado una metodología posible basándose en la gamificación del aprendizaje.

El aumento de artículos científicos relacionados con la gamificación en las aulas de todo el mundo es una realidad. Desde el punto de vista docente, la incorporación de la gamificación supone una forma de fomentar el trabajo en el aula conectando con los intereses de los niños y adolescentes (nativos digitales), además de reforzar la calidad del aprendizaje a través de la interacción social y proyectos proactivos basados en el uso de algo que les resulta tan familiar como son las TIC.

La mayoría de estas soluciones gamificadas incluyen una serie de elementos comunes y consensuados, como son los puntos de experiencia (puntos que los jugadores van acumulando a medida que juegan y que representan su progreso), los niveles, los rankings, las insignias y los retos. También emplean un conjunto de mecanismos eficaces: el uso de las nuevas tecnologías, un feedback continuo que permita a los estudiantes conocer su evolución a lo largo del juego, el uso de hilos narrativos y, finalmente, tener muy claro el tipo de alumnado al cual va dirigida la solución gamificada. También se han detectado, esencialmente, dos debilidades. La primera está relacionada con las dificultades aparecidas en los procesos evaluadores, mientras que la segunda hace referencia a la ausencia de frameworks que faciliten un diseño claro y formal de los juegos.

Como trabajo futuro se prevé implementar, en el nivel experto, problemas de Tecnología Industrial similares a los planteados en las pruebas de acceso a la universidad (PAU) en Cataluña (descritos en la Sección 3) para aumentar la motivación de los alumnos. Además, se propone controlar los niveles de dificultad para que sean incrementales, en el sentido de que primero un alumno debe completar un nivel para llegar al siguiente. Esta configuración permitirá que el alumno sienta que está evolucionando en el juego y su sentimiento de compromiso e implicación sea más elevado y satisfactorio. 


\section{Agradecimientos}

Se quiere agradecer la colaboración prestada por las alumnas y los alumnos de la asignatura Tecnología Industrial de primero y segundo de bachillerato del Institut Quatre Cantons del Poblenou. Esta experiencia docente no habría sido posible sin su ayuda.

Este trabajo ha sido financiado por el proyecto nacional: TIN2013-45303-P “ICT-FLAG: Enhancing ICT education through Formative assessment, Learning Analytics and Gamification".

\section{Referencias}

Baneres, D. (2016). Aprendiendo jugando fundamentos de sistemas digitales. En Actas de las XXII Jornadas de Enseñanza Universitaria de la Informática, Jenui 2016 (pp. 269-276). Universidad de Almería.

Barata, G., Gama, S., Jorge, J., y Gonçalves, D. (2013). Engaging engineering students with gamification. En Games and Virtual Worlds for Serious Applications (VS-GAMES), 2013 5th International Conference on (pp. 1-8). EEUU: IEEE. doi: https://doi.org/10.1109/vs-games.2013.6624228

Caponetto, I., Earp, J., y Ott, M. (2014). Gamification and education: A literature review. En ECGBL 2014: Eighth European Conference on Games Based Learning (pp. 50-57).

Contreras, R. S. (2016). Gamificación en aulas universitarias. Bellaterra: Instituto de la Comunicación, Universitat Autònoma de Barcelona.

Eguía Gómez, J. L., Contreras Espinoza, R. S., y Solano Albajés, L. (2015). Juegos digitales desde el punto de vista de los profesores: Una experiencia didáctica en aulas primaria catalanas. Education in the Knowledge Society (EKS), 16(2), 31-48.

Espinosa, R. S. C., y Gómez, J. L. E. (2012). Videojuegos en dispositivos móviles para desarrollar competencias en alumnos de primaria. En Mobile Communication 2012: Experiències i recerques sobre comunicació mòbil (pp. 56-64). Grup de Recerca d'Interaccions Digitals.

Fadel, L. M., Batista, C., Ulbricht, V. R., y Vanzin, T. (2014). Gamificação na educação. Pimenta Cultural.

García-Peñalvo, F. J. (2016). Proyecto TACCLE3 - Coding. En F. J. García-Peñalvo \& J. A. Mendes (Eds.), XVIII Simposio Internacional de Informática Educativa, SIIE 2016 (pp. 187-189). Salamanca, España: Ediciones Universidad de Salamanca.

Hamari, J., Koivisto, J., y Sarsa, H. (2014). Does gamification work? A literature review of empirical studies on gamification. En 2014 47th Hawaii International Conference on System Sciences (pp. 30253034). EEUU: IEEE. 
Lambruschini, B. B., y Pizarro, W. G. (2015). Tech-Gamification in university engineering education: Captivating students, generating knowledge. En 2015 10th International Conference on Computer Science \& Education (ICCSE) (pp. 295-299). EEUU: IEEE.

Llorens-Largo, F., Gallego-Durán, F. J., Villagrá-Arnedo, C. J., Compañ-Rosique, P., Satorre-Cuerda, R., y Molina-Carmona, R. (2016). Gamification of the Learning Process: Lessons Learned. IEEE Revista Iberoamericana de Tecnologias del Aprendizaje, 17(4), 227-234. doi: http://dx.doi.org/10.1109/ RITA.2016.2619138

Llorens-Largo, F., Villagrá-Arnedo, C. J., Gallego-Durán, F. J., Satorre-Cuerda, R., Compañ-Rosique, P., y Molina-Carmona, R. (2016). LudifyMe: An Adaptative Learning Model Based on Gamification. En S. Caballé y R. Clarisó (Ed.). Formative Assessment, Learning Data Analytics and Gamification in ICT Education. Elsevier-Academic Press. doi: https://doi.org/10.1016/b978-0-12-803637-2.00012-9

Mora, A., Riera, D., González, C., y Arnedo-Moreno, J. (2015). A literature review of gamification design frameworks. En Proceedings of 20157 th International Conference on Games and Virtual Worlds for Serious Applications (VS-Games) (pp. 1-8). EEUU: IEEE. doi: https://doi.org/10.1109/vs-games.2015.7295760

Nebel, S., Schneider, S., y Rey, G. D. (2016). Mining learning and crafting scientific experiments: a literature review on the use of Minecraft in education and research. Journal of Educational Technology \& Society, 19(2), 355-366.

Martín del Pozo, M. (2015). Videojuegos y aprendizaje colaborativo. Experiencias en torno a la etapa de Educación Primaria/Video games and collaborativelearning. Experiences related to Primary Education. Education in the Knowledge Society, 16(2), 69-89. doi: https://doi.org/10.14201/eks20151626989

Rubio, E. P. (2014). Juegos como elemento docente en un entorno TIC. Revista Aequitas: Estudios sobre historia, derecho e instituciones, (4), 407-416.

Sanmugam, M., Abdullah, Z., Mohamed, H., Aris, B., Zaid, N. M., y Suhadi, S. M. (2016). The affiliation between student achievement and elements of gamification in learning science. En Proceedings of 2016 4th International Conference on Information and Communication Technology (ICoICT) (pp. 1-4). EEUU: IEEE. doi: https://doi.org/10.1109/ICoICT.2016.7571962

Toda, A. M., do Carmo, R. S., Silva, A. L., y Brancher, J. D. (2014). Project SIGMA-An Online tool to aid students in Math lessons with gamification concepts. International Conference of the Chilean Computer Science Society (SCCC) (pp. 50-53). doi: https://doi.org/10.1109/sccc.2014.35

Wu, Q., Zhu, Y., y Luo, Z. (2015). A Gamification Approach to Getting Students Engaged in Academic Study. Bulletin of the IEEE Technical Committee on Learning Technology, 17(4), 26-29. 